\title{
El sesgo atencional en los trastornos relacionados con sustancias. Aspectos teóricos, evaluativos y de tratamiento
}

\author{
Attentional bias in substance-related disorders.
}

Theoretical, evaluative and treatment aspects

Rodrigo Moreta-Herrera ${ }^{1}$ y Carlos Reyes-Valenzuela ${ }^{2}$

\begin{abstract}
${ }^{1}$ Candidato a doctor en Psicología Clínica y de la Salud por la Universidad Autónoma de Madrid, España. Máster Universitario en Psicología: individuo, grupo, organización y cultura. Profesor e investigador de la Escuela de Psicología de la Pontificia Universidad Católica del Ecuador, Sede

Ambato. Email: rmoreta@pucesa.edu.ec

${ }^{2}$ Doctor en Psicología Social por la Universidad Autónoma de Madrid. Licenciado en Psicología.

Profesor investigador del programa de Derechos Humanos de la Universidad Andina Simón

Bolívar, Sede Ecuador. Email: carlos.reyes@uasb.edu.ec
\end{abstract}

Facultad de Psicología, Universidad Autónoma de Madrid, Campus Cantoblanco, España.

\section{Resumen}

En la actualidad, existe mayor interés sobre los estudios acerca de la cognición implícita y su función en la conducta. Los modelos explicativos actuales de la psicopatología tienen como limitante la escasa consideración de los procesos cognitivos automáticos en la conducta psicopatológica, como la relacionada con el consumo de sustancias. En este estudio, se revisa al sesgo atencional en los trastornos por consumo y sus implicaciones en la psicopatología, la medición del fenómeno y su aplicación para la intervención. El sesgo atencional hacia las señales críticas de sustancias o drogas es un mecanismo disfuncional de la atención selectiva que opera frecuentemente a nivel automático o implícito y en el que la asignación y focalización de la atención es desproporcionada en el tiempo, entre un estímulo específico (imagen o palabra relacionada con una droga) versus un estímulo neutro. Atrae la atención de la persona al estímulo crítico del que, posteriormente, resulta difícil de desacoplarse y facilita otros procesos cognitivos subsecuentes al consumo. Se presenta evidencia de la incidencia del sesgo atencional en el consumo, el curso, la gravedad, el craving (necesidad intensa de consumo), la abstinencia, la recaída y la probabilidad de fracaso en el tratamiento. Además, explica sobre el fenómeno del consumo, desde la cognición implícita, el inconsciente y la neurociencia cognitiva. Finalmente, en este estudio, se revisan propuestas de intervención sobre los problemas de consumo con técnicas de modificación del sesgo atencional complementarias a los tratamientos cognitivo-conductuales actuales. Se considera necesario explorar sobre esta línea de investigación y su relación con los trastornos por consumo de sustancias dentro de la región.

Palabras clave: atención, cognición, consumo, sesgo, sustancias.

\section{Abstract}

The objective of this work is to investigate attentional bias as an automatic cognitive process and its implications in substance 
use disorders. Cognitive processes are fundamental in the formulation of the different explanatory models about psychopathology. Of the various cognitive processes, attention is a significant determinant, because although it fulfils various activities such as orientation and wakefulness, it is cognitive control that arouses relevant interest for it. This is due to the fact that it fulfills a task of linking between signal uptake and the achievement of other cognitive processes that intervene in the behavioral response mechanism. When a cognitive process like attention is markedly altered it will trigger impaired sequential cognitive functioning, along with the emission of disturbing behavior. Psychology considers that cognitive processes operate consciously (voluntarily) and automatically (implicitly); that is, in a dual way in which these processes such as attention maintain a continuum of coordinated gradual variations that regulate behavior. Failure to consider automatic cognitive processes is a notorious limitation for current explanatory models of psychopathological behavior (including compulsive substance use); given that many of these underlying cognitive processes are often not considered to influence higher mental processes and therefore behavior, without the individual necessarily being aware of this. In fact, this can help to understand pathological behaviors that for the investigator or the professional of psychology can be incomprehensible or paradoxical.

Among the various underlying and altered processes, the attentional bias towards critical signals associated with substances has investigative relevance. Since it is a dysfunctional mechanism of selective attention, which operates frequently at an automatic level and in which the allocation and focus of attention are disproportionate in time, between a specific stimulus (image or word related to a drug) versus a neutral stimulus. And it attracts a person's focus of attention to the critical stimulus, from which it later becomes difficult to decouple. This later facilitates other altered cognitive processes subsequent to consumption. The generation of an attentional bias towards a specific signal has its origin in the association between the signal and the behavior, which can be avoidant (recurrent in phobias) or attractive (frequent in substance or food consumption) and that is conditioned until becoming an automatic process of difficult voluntary control. Although it is true that attention bias may be present in all individuals, the evidence reveals the marked and recurrent presence in various pathologies such as mood disorders (depressive), anxiety, food intake, phobias, of personality among others. And also included in substance use disorders. The findings show that attention bias has an impact on substance use, course, the severity of the disorder, craving, withdrawal, relapse, and the probability of treatment failure. Working with an attentional bias for specific signals on substances can generate difficulty, in the case of evaluation because it is assessed through software that usually measures the eye-hand reaction time or eye movement when faced with neutral and critical signals, which can be a limitation for the usual clinic; while at the intervention level, it is required to formulate bias retraining processes that require continuous exercise as a complementary technique to the usual cognitive-behavioral treatment. It is considered necessary to explore this line of research and its relationship with substance use disorders within the region.

Keywords: attention, cognition, consumption, bias, drugs.

\section{Introducción}

La función de los procesos cognoscitivos (conscientes o automáticos) es fundamental en la formulación de modelos explicativos de los trastornos relacionados con sustancias. La evidencia muestra el papel que juegan los distintos procesos (atención, pensamiento, consciencia, entre otros) en el desarrollo, la consolidación y la mejora de los problemas que conlleva el consumo de sustancias (Field 
y Cox, 2008; Field, Marhe y Franken, 2014; Madoz-Gúrpide y Ochoa-Mangado, 2012; Schoenmakers et al., 2010), por lo que su profundización ayuda a un mejor entendimiento de este grupo de psicopatologías.

Se conoce que los modelos racionalistas imperantes no son suficientes para dar una explicación precisa de la psicopatología, incluida la del consumo (Froufe, Sierra, Zancos y García, 2017). Esto es porque los procesos cognitivos no solo operan de manera deliberada, sino de forma subyacente a la consciencia que modelan una conducta y es en estos procesos no deliberados en los que existe amplio margen explicativo de la psicopatología del consumo (Asmaro, Carolan y Liotti, 2014; Díaz-Batanero et al., 2018).

Dentro de los procesos cognitivos, la atención es determinante para la psicopatología en general $\mathrm{y}$, aunque esta muestra distintas funciones como orientación, alerta y vigilancia, y control cognitivo (Posner y Petersen, 1990), es en esta última -relacionada con el control ejecutivo- que la investigación actual se interesa para explicar la respuesta conductual del consumo. Así, por ejemplo, los hallazgos detallan el incremento a mediano y largo plazo del consumo compulsivo e irrefrenable de sustancias por procesos atencionales alterados o disfuncionales (Carpenter et al., 2012; Field et al., 2016; Sánchez-López et al., 2015).

El no considerar el mecanismo automático de los procesos cognitivos, en especial de la atención en los modelos de explicación y cura, limita las herramientas de intervención y soporte ante las dificultades del consumo y afectan a la eficiencia terminal de los tratamientos actuales. Por esto, el desarrollo de la investigación sobre la cognición inconsciente se torna relevante para su estudio y desarrollo.

\section{Procesos atencionales deliberados y automáticos}

La atención es el mecanismo que filtra la información recibida -estímulos- por el aparato sensorial (Posner y Snyder, 1975; Treisman, 1969) y permite la asignación de recursos cognitivos al procesamiento específico de los estímulos captados (Kahneman, 1973) para emitir una respuesta. A la atención se la cataloga como una función cerebral superior por el elevado número de estructuras cerebrales involucradas y la interacción neuronal que mantiene (Estévez-González, García-Sánchez y Junqué, 1997). Por lo tanto, comprende tanto un mecanismo de control como de regulación de los procesos cognitivos subsecuentes (Ojeda et al., 2002; Roselló, 1998). El desequilibrio en esta función repercute en el resto de los procesos cognitivos posteriores (Field, Marhe y Franken, 2014).

Los procesos atencionales presentan una doble operatividad para desempeñarse, pues presentan un funcionamiento controlado $\mathrm{y}$ automático simultáneo como propiedad (de Paula et al., 2012; Froufe, Sierra, Zancos y García, 2017). A nivel automático, se emplea para favorecer el procesamiento mecánico de captación de estímulos e interacción con el entorno, para lo cual no se requiere de intencionalidad o esfuerzo consciente. Mientras, a nivel voluntario o controlado, se requiere su uso para atender a situaciones más complejas para la solución al no encontrarse sobreatendidas que, por el contrario, requiere de intencionalidad en el acto de focalización de la atención y de esfuerzo consciente para enfocar la atención, lo cual puede interferir con otras funciones cognitivas e interrumpir, incluso, ciertos procesos (Ojeda et al., 2002).

A través de esto, se puede estimar, por una parte, la capacidad dual de la atención para operar a través de una funcionalidad con variaciones graduales coordinadas entre procesos automáticos y deliberados (mas no dicotómica), que actúan con sinergia y equilibrio y pueden ser más eficientes en la administración de recursos (físicos, psicológicos y otros); por otra, la idea de racionalidad limitada, que opera en las actividades cotidianas y en la toma de decisiones de un individuo, que incrementa la posibilidad de errar de manera 
sesgada (Torres-Salazar et al., 2020). Esta modulación de los procesos automáticos y controlados puede verse comprometida si no se logra el equilibrio deseado, lo cual se refleja en procesos cognitivos y respuestas conductuales anormales de la vida cotidiana (Robalino y Musso, 2018) e incluso en la psicopatología (Yeung y Sharpe, 2019) debido a la presencia de deformación o sesgo atencional.

\section{Sesgos atencionales y psicopatología}

Como se mencionó anteriormente, esta dificultad en la modulación de los procesos automáticos y controlados genera un efecto de alteración del funcionamiento correcto de los procesos cognitivos, lo cual desemboca en procesos anormales como son los sesgos cognitivos (Haselton, Nettle y Murray, 2016). En el caso de la atención, el sesgo cognitivo de la atención o Sesgo Atencional (SA) es un mecanismo disfuncional de la atención selectiva en el que la asignación y focalización es desproporcionada en tiempo ante un estímulo específico (palabra, imagen, otros) frente a un estímulo neutral (Marks, Pike, Stoops y Craig, 2015). Además, este fenómeno activa, específicamente, otros procesos cognitivos alterados o sesgados subsecuentes. Así por ejemplo, un SA focalizado en estímulos relacionados con las drogas (estímulo crítico) en personas con trastornos por consumo desencadena pensamientos favorables a él, craving (necesidad intensa de consumo), predisposición y ejecución de la misma conducta de consumo (Cox et al., 2014). De este modo, se incrementa el riesgo y la vulnerabilidad al consumo, a diferencia de aquella persona que no muestra SA.

El SA tiene un carácter de proceso alterado automático, que se consolida por: (a) la estimulación externa (Ojeda et al., 2002); (b) la práctica; y (c) la repetición, que ayudan en la mejora de la velocidad de respuesta a la captación de estímulos críticos -asociacionismo(Posner y Snyder, 1975). Además, es un mecanismo con un fin adaptativo al contexto (sea equívoco o adecuado). Esta captación rápida, espontánea y preferencial del estímulo crítico -por condicionamiento-, se superpone a otros estímulos -neutros- (Wiers et al., 2013) y cambia la dinámica atencional. Esto es porque la asociación estímulo-respuesta atencional guardan un contenido emocional, que permite interpretar a los estímulos críticos como amenazantes -respuesta evitativa- o placenteros -respuesta atractiva- y favorece la consecución de procesos cognitivos en concordancia a dicho contenido. Los estímulos críticos y la preferencia por su selección configuran no solo la práctica de la conducta, sino también su vivencia e interpretación interna (Gladwin, 2017) de modo que este circuito de retroalimentación positiva a futuro ayuda a consolidar un sistema de respuesta de patrones conductuales recurrentes automáticos.

Para que se desarrolle una atención focalizada normal hacia un estímulo son necesarios: (a) la detección de estímulos; (b) la discriminación entre estímulos de interés e irrelevantes; y (c) el acoplamiento al estímulo crítico (Posner y Snyder, 1975; Posner y Petersen, 1990). Pero en el caso del SA, la detección de los estímulos críticos es más rápida de lo usual, la discriminación es preferencial y el desacoplamiento del estímulo o el cambio hacia otro es dificultoso (MacLeod et al., 2002), por lo que se desencadena un proceso de fijaciones innecesarias, improductivas y no beneficiosas en el individuo. Incluso, debido a la consecución de otros procesos cognitivos subsecuentes, el desacoplamiento a los estímulos críticos falla por el aparecimiento del pensamiento rumiativo (Bravo, Pearson y Henson, 2016).

Este fenómeno del SA se ha identificado en distintos procesos psicológicos del individuo, incluida la psicopatología (Cox, Fadardi, Intriligator y Klinger, 2014; Field et al., 2016; Franken, 2007; McLeod, 2002). La evidencia muestra la presencia del SA en trastornos depresivos (Duque, López-Gómez, Blanco y Vázquez, 2015; Sánchez y Vázquez, 2012), ansiógenos (Hadwin y Richards, 2016; Mac Leod et al., 2002), fóbicos (Rosa, Esteves y Arriaga, 2015), de personalidad (Begh et al., 
2015), de la conducta alimentaria (Schmitz y Svaldi, 2017) y en las drogodependencias (Díaz-Batanero et al., 2018; Field y Cox, 2008; Field, Marhe y Franken, 2014; Schoenmakers et al., 2010). Si bien requiere su análisis y seguimiento en este último caso sobre los problemas con sustancias, aún la evidencia es escasa y arroja resultados contradictorios que requieren de una revisión profunda y sistematizada.

\section{Sesgo atencional en los trastornos}

\section{por consumo de sustancias}

Un trastorno por consumo de sustancias generalmente suele ser identificado por los niveles elevados de craving y la práctica compulsiva e irrefrenable del consumo, a pesar del grado de conciencia que se tiene de las consecuencias negativas que provocan (Stacy y Wiers, 2010). Según la evidencia actual, la práctica del consumo compulsivo de alcohol y otras sustancias se ha visto incrementada en los últimos años, en especial en grupos vulnerables como los adolescentes (Moreta-Herrera et al., 2020).

La propuesta cognitiva ante este fenómeno explica que esta condición está dada por mecanismos y esquemas mentales que operan a modo de cognición implícita que llevan al individuo, de manera automática $o$ con consciencia limitada, hacia la ejecución de la conducta de consumo.

Dentro de estos mecanismos automáticos que marcan la conducta compulsiva, se encuentran los procesos atencionales alterados o sesgados, que se producen cuando estímulos críticos - contenido o significado asociado con una sustancia- captan significativamente la atención del sujeto hacia dicho estímulo, en preferencia a otros estímulos (Sánchez-López, Quinto-Guillen, Pérez-Lucas y Jurado-Barba, 2015) y producen mecanismos subsecuentes que conllevan a la conducta en mención.

Cabe señalar que tanto las vías neuronales como las áreas cerebrales de recompensa ante el consumo son diferentes en varios aspectos según la sustancia que se ingiere, por lo que no se puede estimar que los trastornos por consumo de sustancias sean iguales en todos los casos (Begh et al., 2015). Así, la presencia de un SA hacia una sustancia específica responde mayoritariamente a la sustancia de consumo habitualizada o condicionada, por lo que no es viable considerar que la existencia de un SA hacia estímulos específicos desencadene una conducta de consumo de cualquier sustancia en general, pues previamente se requiere de la asociación entre la sustancia, el efecto y el estímulo. En situaciones como las politoxicomanías (e. g., cocaína y alcohol, entre otras) es esperado, desde luego, que ocurra más de un SA (Díaz-Batanero et al., 2018; Marks, Pike, Stoops y Craig, 2015).

Los estudios sobre los SA señalan que el curso, el desarrollo y el mantenimiento de una conducta de consumo dependen de los estímulos a los que se exponen los consumidores y al tipo de procesamiento atencional (SánchezLópez et al., 2015). La captación preferencial de la atención del consumidor de señales críticas aumenta en importancia como resultado de un aprendizaje pavloviano (Robinson y Berridge, 1993) asociado al consumo, incluso desde condiciones no compulsivas en el consumo necesariamente; por ejemplo, en el consumo social empedernido de alcohol ya se detecta el aparecimiento del SA (Pennington et al., 2020). Es decir que las señales críticas se vuelven más destacadas a través del aprendizaje iniciado y sostenido por emparejamiento repetido con las sustancias como recompensa (Franken, 2007). Además, la presencia, la intensidad y la permanencia del SA está relacionada con la cantidad habitual de consumo de una sustancia (Cox, Fadardi y Pothos, 2006), y también en su deprivación (Freeman, Morgan, Beesley y Curran, 2012) cuando el consumo ya se consolidó.

Desde la neurofisiología de las adicciones, las neuroadaptaciones dopaminérgicas frontolímbicas constituyen procesos sensibilizados a los incentivos de las conductas de consumo 
y son una fuente subyacente a las señales externas asociadas a las sustancias (Wiers et al., 2015). También, la mediación por el incremento en la liberación de la dopamina en el núcleo de recompensa de las estructuras cerebrales (Goldstein et al., 2007) influye en la generación de la asociación entre estímulo externo y respuesta fisiológica. Así, el efecto gratificante anticipado de las sustancias a través de la búsqueda de señales crea en el consumidor una mayor participación efectiva y le encamina a la experiencia subjetiva del deseo (craving) de consumir. Con posterioridad, estas señales generan respuestas motivacionales en los consumidores, los cuales son disparados relativamente de manera automática (Wiers, Bartholow y van den Wildenberg, 2007).

\section{Diferencias del SA entre consumidores y no consumidores}

Los hallazgos previos señalan la existencia de diferencias del SA entre personas con situación de consumo patológico que de aquellas que no lo tienen o que no consumen (Cox, Brown y Rowlands, 2003; SánchezLópez et al., 2015; Zack et al., 2001) y que influyen a nivel de captación, procesamiento y ejecución de actividades. La captación de señales específicas y el atractivo que causan tienen mayor implicación entre quienes tienen habitualizado el consumo frente a aquellos que no lo tienen. Tales hallazgos son observados en dependendientes del alcohol (Field y Cox, 2008; Jones, Bruce, Livingstone y Reed, 2006), el tabaco (Begh et al., 2015; Munafo et al., 2003), la heroína (Lubman et al., 2000), la cocaína (Carpenter et al., 2012; Vadhan et al., 2007), las metanfetaminas (Dean et al., 2019).

A nivel de procesamiento de la información captada en aquellos con consumo patológico, el procesamiento de la información crítica recibida tarda más tiempo debido al acoplamiento con dichos estímulos que en los segundos (Cox et al., 2002; Cox, Brown y Rowlands, 2003; Sánchez-López et al., 2015). Así por ejemplo, se reporta en consumidores de tabaco versus abstemios con evaluación a través del Color Stroop Test que tardaron más tiempo en responder con el color en una palabra con contenido crítico que en palabras con contenido neutro, mientras que a nivel de ejecución de actividades se evidencia que existe mayor afectación (Field y Cox, 2008). En consumidores de cannabis se detectó que cometen más errores de acierto frente a estímulos con información crítica que los no consumidores (Asmaro, Carolan y Liotti, 2014); también se encontró rendimiento de atención más pobre en consumidores versus no consumidores (Alcorn III, 2019). Lo mismo sucedió en consumidores de alcohol social empedernido, quienes mostraron una búsqueda visual de objetivos críticos más rápida que aquellos que no consumen o consumen poco (Pennington et al., 2020; Qureshi et al., 2019). Al parecer, estos hallazgos permiten identificar no solo la tendencia involuntaria en los consumidores de dirigir los recursos atencionales, sino el uso de procesos congnitivos automáticos e inconscientes subsecuentes que operan con este tipo de estímulos.

\section{Efectos del sesgo atencional en los trastornos relacionados con el consumo de sustancias}

De manera general y según la evidencia, el SA tiene un efecto causal en los procesos de conducta abusiva, dependencia y sostenimiento de la práctica del consumo (Weinstein y Cox, 2006). A más de ello exacerba el patrón de consumo haciéndolo más intenso (Field y Eastwood, 2005). Influye también directamente en los niveles de gravedad de la dependencia a sustancias (Bearre, Sturt, Bruce y Jones, 2007; Fadardi y Cox, 2008), en la eficiencia terminal de los tratamientos del que es un predictor negativo (Carpenter, Schreiber, Church y McDowell, 2006; Carpenter et al., 2012; Bardeen et al., 2014) e incluso, en el incremento de la probabilidad de la recaída, aun con largos períodos de abstinencia (Begh 
et al., 2015; Carpenter et al., 2006; Cox et al., 2002; Dean et al., 2019; Heinz, Beck y Grüsser, 2009; Powell et al., 2010).

En aspectos específicos de los trastornos por consumo de sustancias también hay incidencia del SA en el deseo por consumir (craving) por ejemplo, el cual se incrementa tras la exposición de un consumidor a estímulos críticos (Pennington et al., 2020). Para ciertos autores la relación entre el SA y el craving se encuentra mediada tanto por el uso como por la habituación de la sustancia (Field, Munafo y Franken, 2009), aunque esta opinión no es compartida por todos (Franken, 2003). Se conoce por estudios de neuroimagen que pacientes condicionados, al estar expuestos a señales específicas sobre sustancias, muestran mayor activación cerebral en las áreas mesolímbicas relacionadas con el refuerzo (Heinz, Beck y Grüsser, 2009), el craving (Heinz et al., 2005) y la recompensa (Park et al., 2011). Este fenómeno, al parecer, actúa en consumidores de cannabis (Asmaro, Carolan y Liotti, 2014), tabaco (Powell et al., 2010) y alcohol (Field y Cox, 2008). De esta manera, el efecto del SA en los trastornos por consumo es significativo, pues comprende un elemento importante en la dinámica del consumo coumpulsivo que, al no ser atendido adecuadamente en los tratamientos, constituye un factor de riesgo para la complicación del trastorno.

\section{Medición y modificación del sesgo atencional}

La valoración del SA en el proceso de diagnóstico y tratamiento de distintas patologías se realiza por medio de tareas específicas en las que se evalúa generalmente el tiempo de respuesta ante la exposición a estímulos críticos y neutros para así inferir la presencia o no del sesgo. Una de las evaluaciones más conocidas es la derivada del test clásico de verbalización del color en grupos de palabras críticas y neutras, el Color Stroop Test (Stroop, 1935). En el caso del SA, se comparan los tiempos de respuesta al nombrar el color de una palabra crítica frente a una neutra. Se considera que las personas que presentan problemas con el consumo de alcohol, tabaco u otra sustancia tardarán más tiempo en nombrar el color de la palabra crítica que de aquellas irrelevantes. En este tipo de evaluaciones se intenta valorar el funcionamiento del lóbulo frontal (Reeve y Schandler, 2001), la flexibilidad cognitiva (Fisher, Freed y Corkin, 1990) y la capacidad de inhibición de los sujetos (Milham, Banich y Barad, 2003). Valoraciones de este tipo son probadas para medir el SA en alcohol con variantes especiales (Alcohol Stroop Test) (Cox, Brown y Rowlands, 2003; Cox et al., 2006; Sánchez-López et al., 2015); tabaco (Calleja y Hernández-Pozo, 2009; Munafo et al., 2003) y cocaína (Díaz-Batanero et al., 2018).

Otra de las técnicas es la de sondeo visual de puntos o dot-probetas $k$ (MacLeod et al., 2002), que consiste en identificar en una pantalla de ordenador lo más rápido posible una sonda o señal (punto u otro) que aparece tras la ubicación de imágenes a modo de estímulos (crítico o neutro). El SA ocurre en las respuestas más rápidas a las sondas o señales que reemplazan los estímulos críticos $\mathrm{y}$ sugiere que el participante consumidor involucra más recursos cognitivos ante este tipo de señales. Estudios de este tipo se han aplicado para la medición del SA hacia el tabaco en fumadores versus no fumadores (Bradley, Field, Mogg y De Houwer, 2004), alcohol (Gladwin, 2017; Pennington et al., 2020) y en otras condiciones clínicas (Price et al., 2015).

En cuanto a la modificación del SA, dado que las técnicas de medición generan un esfuerzo y una actividad práctica para su resolución, el uso continuado y repetitivo de estas acciones de selección e identificación a través de las tareas es considerado positivo para el desarrollo de técnicas de modificación o corrección de este (Hakamata et al., 2010). La modificación del SA, en este caso, conforma un proceso artificial para inhibir el proceso de captación y acoplamiento de las señales críticas e irrumpir en los mecanismos cogni- 
tivos subyacentes. Así, se busca trabajar en la automatización de los procesos atencionales para producir cambios cognitivos de bajo nivel, pues a menudo estos son inaccesibles desde la conciencia (Froufe, 2017; Wadlinger e Isaacowitz, 2010). Igualmente, se trabaja en el fortalecimiento del control de los procesos atencionales (Houben, Wiers y Jansen, 2011) mediante el cambio de las tendencias de los sesgos de atención y la acción posterior (Wiers et al., 2013).

Se estima que la modificación del SA también puede ser utilizado como una técnica coadyuvante de modificación de la conducta en el tratamiento de los trastornos relacionados con sustancias, mas no del reemplazo de terapéuticos como la terapia cognitivo-conductual, por ejemplo. El entrenamiento o reentrenamiento en el SA daría más peso en los programas habituales de intervención, porque el SA juega un rol en la falta de control del comportamiento (Cox, Fadardi y Klinger, 2014), aunque no se puede aplicar para todos los tipos de trastorno por consumo de sustancias. El seguimiento a los SA podría permitir la predicción de la situación del consumo de sustancias a futuro (e.g. en el postratamiento), aunque la evidencia sobre esto aún no es consistente (Dean et al., 2019; Field, Marhe y Franken, 2014).

Con estos entrenamientos se pueden alterar las contingencias de aparición de los estímulos, a fin de forzar de modo automático la atención del participante hacia un patrón atencional específico adaptativo (Duque et al., 2015) y provocar un efecto distinto al ya condicionado. Desde un punto de vista neurológico, la efectividad de la modificación del sesgo afecta la actividad del área mesolímbica de las señales inducidas y una reducción en la reactividad neuronal, la cual es clave para el entendimiento del mecanismo de la efectividad terapéutica del entrenamiento (Wiers et al., 2015).

Es necesario anotar que la efectividad de los entrenamientos de modificación de SA requieren de tres aspectos para su adecuado desarrollo: (a) motivación del individuo para mejorar el rendimiento de la formación y el control de su atención; (b) presentación de un gran número de estímulos durante el tratamiento, desde estímulos generalizados hasta nuevos estímulos que solo han sido encontrados después de tratamientos; y (c) realizar tratamientos de múltiples sesiones, pues hay más efectos prolongados que aquellos con sesiones únicas (Schoenmakers et al., 2010).

\section{Implicaciones del estudio del}

\section{sesgo atencional en los trastornos \\ relacionados con sustancias}

Desde el punto de vista teórico, el estudio del SA en los trastornos relacionados con el consumo de sustancias resulta significativo por su contribución a una nueva interpretación de la psicopatología, que toma los avances actuales sobre la cognición implícita y el inconsciente cognitivo, pues gran parte de los procesos cognitivos trabajan procesando información que muchas veces no llega a la consciencia (Froufe, 2000). Este hecho está fuertemente involucrado en la psicopatología, pero no se lo identifica adecuadamente. Así, el SA representaría un síntoma relevante en la psicopatología del consumo, pero que resulta irrelevante para la psicología moderna. De por sí se debe considerar que el sesgar, aunque puede ser contradictorio, es un recurso que cumple ciertas funciones en el ambiente para el individuo, como sostener conductas patológicas (como las ya mencionadas) o también conductas de salud y bienestar (Torres-Salazar et al., 2020).

Por otra parte, la idea de la presencia de mecanismos implícitos y automáticos que subyacen a la conciencia desestima la creencia de que las conductas adictivas resultan de un proceso irreflexivo y desorganizado $\mathrm{y}$ que, por el contrario, se activan espontáneamente durante los procesos críticos de toma de decisiones (Stacy y Wiers, 2010) (e. g. 
deseo de consumo y ejecución ante una necesidad intensa). Con esto se construye una fuerte crítica a los modelos racionalistas que consideran que la mecánica del consumo se da por procesos más conscientes como las actitudes favorables o desfavorables frente al consumo (Moreta-Herrera, Mayorga-Lascano, León-Tamayo e Ilaja-Verdesoto, 2018) o en la toma de decisiones, incluso cuando los peligros a largo plazo del uso continuado de drogas son ampliamente conocidos por el consumidor (Wiers et al., 2015). De ahí que esto puede ayudar a entender el comportamiento del consumidor que suele presentarse muchas veces como incomprensible e, incluso, paradójico.

Por otra parte, desde los aspectos relevantes de la psicología básica y experimental, existe también un carácter significativo de los SA en las conductas de consumo, pues la presencia del SA permite detectar con más facilidad y velocidad los estímulos que hay en el ambiente (esto supone un problema si se encuentran en el proceso de evitación o retirada del consumo), estimula respuestas condicionadas o compensatorias, incrementa el deseo por consumir la sustancia o activa patrones de conducta automáticos que conducen a su consumo, y altera el estado emocional de las personas que intentan mantenerse abstinentes e interfiere con sus actividades diarias (Waters y Feyerabend, 2000).

En lo que respecta a la intervención de los trastornos del consumo de sustancias, la inclusión de técnicas de modificación del SA permite ampliar el abanico de técnicas de intervención, pues aumenta también el número de campos de acción que, generalmente, no tienden a ser considerados. La evidencia muestra, incluso, diferencias en drogodependientes que llevaron a cabo tratamientos exitosos con modificación del SA versus tratamientos fracasados en los que no se incluyó la modificación (Cox et al., 2002) pues, al parecer, la probabilidad de éxito del tratamiento es tres veces mejor cuando se presentan bajos niveles de SA (Cox, Pothos y Hosier, 2007). La modificación del SA puede mejorar la eficiencia terminal de los tratamientos debido a que los programas de intervención cognitivo-conductual se encuentran dirigidos hacia los mecanismos de procesamiento voluntario de la información, omitiendo las potenciales desventajas de los procesos involuntarios inmersos en las psicopatologías (McNally, 1995). Además, es un factor relevante en la recaída (Powell et al., 2010; Begh et al., 2015).

Finalmente, como se mencionó antes, las implicaciones de la continuidad sobre el estudio del SA tanto a nivel descriptivo como de intervención permitirán extender el entendimiento de los trastornos por consumo de sustancias, cambiar los paradigmas de explicación y desarrollar estrategias de intervención que sean más efectivas, pertinentes, abaraten costos y puedan ser extendidos a grupos vulnerables que, hoy en día, pueden tener dificultades para acceder a un tratamiento. Es importante señalar que, en el contexto latinoamericano, la exploración de esta línea de investigación cuenta con investigación limitada y no consolidada, en especial en cuanto a los SA dirigidos a sustancias, por lo que se presenta un retraso significativo en el desarrollo de nuevos y mejores tratamientos de intervención basados en los avances de esta línea.

\section{Referencias}

Alcorn III, J. L., Marks, K. R., Stoops, W. W., Rush, C. R. y Lile, J. A. (2019). Attentional bias to cannabis cues in cannabis users but not cocaine users. Addictive Behaviors, 88, 129-136. https://doi.org/10.1016/j.addbeh.2018.08.023

Asmaro, D., Carolan, P. L. y Liotti, M. (2014). Electrophysiological evidence of early attentional bias to drug-related pictures in chronic cannabis users. Addictive Behavoirs, 39, 114-121. https://doi.org/10.1016/j. addbeh.2013.09.012

Bardeen, J. R., Dixon-Gordon, K. L., Tull, M. T., Lyons, J. A. y Gratz, K. L. (2014). An inves- 
tigation of the relationship between borderline personality disorder and cocaine-related attentional bias following trauma cue exposure: The moderating role of gender. Comprehensive Psychiatry, 55, 113-122. https://doi. org/10.1016/j.comppsych.2013.08.011

Bearre, L., Sturt, P., Bruce, G. y Jones, B. (2007). Heroin-related attentional bias and monthly frequency of heroin use are positively associated in attenders of a harm reduction service. Addictive Behaviors., 32, 784-792. https://doi. org/10.1016/j.addbeh.2006.06.019

Begh, R., Munafò, M. R., Shiffman, S., Ferguson, S. G., Nichols, L., Mohammed, M. A., .. . Aveyard, P. (2015). Lack of attentional retraining effects in cigarette smokers attempting cessation: A proof of concept double-blind randomised controlled trial. Drug and Alcohol Dependence, 149, 158-165. https://doi.org/10.1016/j. drugalcdep.2015.01.041

Bradley, B., Field, M., Mogg, K. y De Houwer, J. (2004). Attentional and evaluative biases for smoking cues in nicotine dependence: component processes of biases in visual orienting. Behavioural Pharmacology, 15, 29-36. https:// doi.org/10.1097/00008877-200402000-00004

Bravo, A. J., Pearson, M. R. y Henson, J. M. (2016). Drinking to Cope With Depressive Symptoms and Ruminative Thinking: A Multiple Mediation Moel Among College Students. Substance Use y Misuse, 52(1), 52-62. https://doi.org/10. 1080/10826084.2016.1214151

Calleja, N. y Hernández-Pozo, M. D. (2009). Prueba Stroop computarizada de riesgo tabáquico para adolescentes. Revista Mexicana de Análisis de la Conducta, 35(2), 91-107. http:// www.scielo.org.mx/scielo.php?script=sci_ arttext\&pid=S0185-45342009000200007\&ln$\mathrm{g}=\mathrm{es} \& \mathrm{nrm}=$ iso

Carpenter, K., Martinez, D., Vadhan, N., BarnesHolmes, D. y Nunes, E. (2012). Measures of attentional bias and relational responding are associated with behavioral treatment outcome for cocaine dependence. The American Journal of Drug and Alcohol Abuse, 38, 146-154. https://doi.org/10.3109/00952990.2011.643 986
Carpenter, K., Schreiber, E., Church, S. y McDowell, D. (2006). Drug Stroop performance: relationships with primary substance of use and treatment outcome in a drug-dependent outpatient sample. Addictive Behaviors., 31, 174-181. https://doi.org/10.1016/j. addbeh.2005.04.012

Cox, W. M., Brown, M. A. y Rowlands, L. J. (2003). The effects of alcohol cue exposure on non-dependent drinkers' attentional bias for alcohol-related stimuli. Alcohol and Alcoholism, 38, 45-49. https://doi.org/10.1093/ alcalc/agg010

Cox, W. M., Fadardi, J. S., Intriligator, J. M. y Klinger, E. (2014). Attentional bias modification for addictive behaviors: clinical implications. CNS Spectrums, 215-224. https://doi. org/10.1017/S1092852914000091

Cox, W., Fadardi, J. y Pothos, E. (2006). The addiction-Stroop test: theoretical considerations and procedural recommendations. Psychological Bulletin, 132(3), 443-476. https://doi. org/10.1037/0033-2909.132.3.443

Cox, M., Hogan, L., Kristian, M. y Race, J. (2002). Alcohol attentional bias as a predictor of alcohol abusers' treatment outcome. Drug and Alcohol Dependence, 68, 237-243. https:// doi.org/10.1016/S0376-8716(02)00219-3

Cox, W., Pothos, E. y Hosier, S. (2007). Cognitive-motivational predictors of excessive drinkers' success in changing. Psychopharmacology 192, 499-510. https://doi.org/10.1007/ s00213-007-0736-9

Dean, A. C., Nurmi, E. L., Moeller, S. J., Nader, A., Rozenman, M., Ghahremani, M., Berbeyan, R., ..., London, E. (2019). No effect of attentional bias modification training in methamphetamine users receiving residential treatment. Psychopharmacology, 236, 709-721. https:// doi.org/10.1007/s00213-018-5100-8

de Paula, J. J., de Souza Costa, D., de Moraes, E. N., Nicolato, R., Sedó, M. y Malloy-Diniz, L. F. (2012). Automatic and controlled attentional processes in amnestic mild cognitive impairment: the use of a mini-verbal test. Psychology, 3(5), 379-383. https://doi.org/10.4236/ psych.2012.35053 
Díaz-Batanero, C., Domínguez-Salas, S., Moraleda, E., Fernández-Calderón, F. y Lozano, O. M. (2018). Attentional bias toward alcohol stimuli as a predictor of treatment retention in cocaine dependence and alcohol user patients. Drug and Alcohol Dependence, 182, 40-47. https://doi.org/10.1016/j. drugalcdep.2017.10.005

Duque, A., López-Gómez, I., Blanco, I. y Vázquez, C. (2015). Modificación de Sesgos Cognitivos (MSC) en depresión: Una revisión crítica de nuevos procedimientos para el cambio de sesgos cognitivos. Terapia Psicológica, 33(2), 103-116. https://doi.org/10.4067/ S0718-48082015000200005

Estévez-González, A., García-Sánchez, C. y Junqué, C. (1997). La atención: una compleja función cerebral. Revista de Neurología, 25(148), 1989-1997. https://doi.org/10.33588/ m.25148.97483

Fadardi, J. S. y Cox, W. M. (2008). Alcohol-attentional bias and motivational structure as independent predictors of social drinkers' alcohol consumption. Drug and Alcohol Dependence, 97(3), 247-256. https://doi.org/10.1016/j. drugalcdep.2008.03.027

Field, M. y Cox, W. (2008). Attentional bias in addictive behaviors: a review of its development, causes, and consequences. Drug Alcohol Dependence, 97(1-2), 1-20. https://doi. org/10.1016/j.drugalcdep.2008.03.030

Field, M. y Eastwood, B. (2005). Experimental manipulation of attentional bias increases the motivation to drink alcohol. Psychopharmacology 183, 350-357. https://doi.org/10.1007/ s00213-005-0202-5

Field, M., Marhe, R. y Franken, I. H. (2014). The clinical relevance of attentional bias in substance use disorders. CNS spectrums, 19(3), 225-230. https://doi.org/10.1017/ S1092852913000321

Field, M., Munafo, M. y Franken, I. (2009). A meta-analytic investigation of the relationship between attentional bias and subjective craving in substance abuse. Psychological Bulletin, 135(4), 589-607. https://doi. org/10.1037/a0015843
Field, M., Werthmann, J., Franken, I., Hofmann, W., Hogarth, L. y Roefs, A. (2016). The role of attentional bias in obesity and addiction. Health Psychology, 35(8), 767-780. https://doi. org/10.1037/hea0000405

Fisher, L., Freed, D. M. y Corkin, S. (1990). Stroop color-word test perfomance in patients with Alzheimer's Disease. Journal of Clinical Experimental Neuropsychology, 12, 745-758. https://doi.org/10.1080/01688639008401016

Franken, I. (2003). Drug craving and addiction: integrating psychological and neuropsychopharmacological approaches. Progress in Neuropsychopharmacology and Biological Psychiatry, 27(4), 563-579. https://doi. org/10.1016/S0278-5846(03)00081-2

Franken, I. (2007). Craving, cue-reactivity, attentional bias and relapse in addiction. European Neuropsychopharmacology, 17, S214. https:// doi.org/10.1016/s0924-977x(07)70274-2

Freeman, T., Morgan, C., Beesley, T. y Curran, H. (2012). Drug cue induced over- shadowing: selective disruption of natural reward processing by cigarette cues amongst abstinent but not satiated smokers. Psychological Medicine, 42, 1341. https://doi.org/10.1017/ S0033291711001139

Froufe, M. (2000). Inconsciente Cognitivo: La Mente Oculta. Psikhe, Revista de la Escuela de Psicología, 9(1), 3-11. http://ojs.uc.cl/index. php/psykhe/article/view/20963

Froufe, M., Sierra, B., Zancos, Y. y García, P. (2017). Consciousness, Unconscious Cognition, and their Alterations: Implications of the Dual Mind Model in Psychopathology. Chaos and Complexity Letters, 11(3), 327-346.

Gladwin, T. E. (2017). Attentional bias variability and cued attentional bias for alcohol stimuli. Addiction Research y Theory, 25(1), 32-38. https://doi.org/10.1080/16066359.2016.1196 674

Goldstein, R. Z., Tomasi, D., Rajaram, S., Cottone, L. A., Zhang, L., Maloney, T., ... y Volkow, N. D. (2007). Role of the anterior cingulate and medial orbitofrontal cortex in processing drug cues in cocaine addiction. Neuroscience, 144(4), 1153-1159. https://doi.org/10.1016/j. 
neuroscience.2006.11.024

Hadwin, J. y Richards, J. (2016). Working Memory Training and CBT Reduces Anxiety Symptoms and Attentional Biases to Threat: A Preliminary Study. Frontiers in Psychology, 7(47), 1-12. https://doi.org/10.3389/fpsyg.2016.00047

Hakamata, Y., Lissek, S., Bar-Haim, Y., Britton, J. C., Fox, N. A., Leibenluft, E. y Pine, D. S. (2010). Attention bias modification treatment: A meta-analysis toward the establishment of novel treatment for anxiety. Biological Psychiatry, 68, 982-990. https://doi.org/10.1016/j. biopsych.2010.07.021

Haselton, M. G., Nettle, D. y Murray, D. R. (2016). The evolution of cognitive bias. En D. Buss (Ed). The handbook of evolutionary psychology (second edition) (pp. 1-20). John Wiley \& Sons, Inc.

Heinz, A., Beck, A. y Grüsser, S. (2009). Identifying the neural circuitry of alcohol craving and relapse vulnerability. Addiction Biology 14, 108-118. https://doi.org/10.1111/j.13691600.2008.00136.x

Heinz, A., Siessmeier, T., Wrase, J. et al. (2005). Correlation of alcohol craving with striatal dopamine synthesis capacity and D2/3 receptor availability: a combined [18F] DOPA and [18F] DMFP PET study in detoxified alcoholic patients. American Journal of Psychiatry, 162, 1515-1520. https://doi.org/10.1176/appi. ajp.162.8.1515

Houben, K., Wiers, R. W. y Jansen, A. (2011). Getting a grip on drinking behavior: Training working memory to reduce alcohol abuse. Psychological Science, 22, 968-975. https:// doi.org/10.1177/0956797611412392

Jones, B., Bruce, G., Livingstone, S. y Reed, E. (2006). Alcohol-related attentional bias in problem drinkers with the flicker change blindness paradigm. Psychology Addictive Behaviors, 20, 171-177. https://doi. org/10.1037/0893-164X.20.2.171

Kahneman, D. (1973). Attention and effort. Englewood Cliffs, NJ: Prentice-Hall.

Lubman, D., Peters, L., Mogg, K., Bradley, B. y Deakin, J. (2000). Attentional bias for drug cues in opiate dependence. Psychological Medi- cine, 30, 169-175. https://doi.org/10.1017/ S0033291799001269

MacLeod, C., Rutherford, E., Campbell, L., Ebsworthy, G. y Holker, L. (2002). Selective attention and emotional vulnerability: assessing the causal basis of their association through the experimental manipulation of attentional bias. Journal Abnormal Psychology, 111, 107-123. https://psycnet.apa.org/doi/10.1037/0021843X.111.1.107

Madoz-Gúrpide, A. y Ochoa-Mangado, E. (2012). Alteraciones de funciones cognitivas y ejecutivas en pacientes dependientes de cocaína: estudio de casos y controles. Revista de Neurología, 54(4), 199-208. https://doi.org/10.33588/ m.5404.2011174

Marks, K., Pike, E., Stoops, W. y Craig, R. (2015). The Magnitude of Drug Attentional Bias is Specific to Substance Use Disorder. Psychology of Addictive Behaviors, 29(3), 690-695. https:// doi.org/10.1037\%2Fadb0000084

McNally, R. (1995). Automaticity and the anxiety disorders. Behavorial Research \& Therapy, 33, 747-754. https://doi.org/10.1016/00057967(95)00015-P

Milham, M. P., Banich, M. T. y Barad, V. (2003). Competition for priority in processing increases prefrontal cortex's involvement in top - down: an event - related fMRI study of the stroop task. Cognitive Brain Research, 17, 212-222. https://doi.org/10.1016/S09266410(03)00108-3

Moreta-Herrera, R., Mayorga-Lascano, P., León-Tamayo, A. e Ilaja-Verdesoto, B. (2018). Consumo de sustancias legales, ilegales y fármacos en adolescentes y factores de riesgo asociados a la exposición reciente. Health and Addictions / Salud y Drogas, 18(1), 39-50. https://doi.org/10.21134/haaj.v18i1.333

Moreta-Herrera, R., Rodas, J. A y Lara-Salazar, M. (2020). Factor Validity of Alcohol Use Disorders Identification Test (AUDIT) Using Robust Estimations in Ecuadorian Adolescents. Alcohol and Alcoholism, (on line first). https://doi.org/10.1093/alcalc/agaa126https:// doi.org/10.1093/alcalc/agaa126

Munafo, M., Mogg, K., Roberts, S., Bradley, B. 
y Murphy, M. (2003). Selective processing of smoking-related cues in current smokers, ex-smokers and never- smokers on the modified Stroop task. Journal of Psychopharmacology, 17, 310-316. https://doi. org/10.1177/02698811030173013

Ojeda, N., Ortuño, F., López, P., Arbizu, J., Martí-Climent, J. y Cervera-Enguix, S. (2002). Bases neuroanatómicas de la atención mediante PET-15O: el papel de la corteza prefrontal y parietal en los procesos voluntarios. Revista de Neurología, 35(6), 501-507. https://doi. org/10.33588/rn.3506.2002158

Park, S., Kahnt, T., Rieskamp, J. y Heekeren, H. (2011). Neurobiology of value integration: when value impacts valuation. Journal of Neuroscience, 31, 9307-9314. https://doi. org/10.1523/JNEUROSCI.4973-10.2011

Pennington, C. R., Shaw, D. J., Adams, J., Kavanagh, P., Reed, H., Robinson, M., ...y White, H. (2020). Where's the wine? Heavy social drinkers show attentional bias towards alcohol in a visual conjunction search task. Addiction, 115(9), 1650-1659. https://doi.org/10.1111/ add. 14997

Posner, M. y Petersen, S. (1990). The attention system of the human brain. Annual Review of Neuroscience, 13, 25-42. https://www. annualreviews.org/doi/10.1146/annurev. ne.13.030190.000325

Posner, M. y Snyder, C. (1975). Facilitation and inhibition in the processing of signals. En P. Rabbit y S. Dornis (Eds.), Attention and performance. New York: Academic Press.

Powell, J., Dawkins, L., West, R., Powell, J. y Pickering, A. (2010). Relapse to smok- ing during unaided cessation: clinical, cognitive and motivational predictors. Psychopharmacology, 212, 537-549. https://doi.org/10.1007/ s00213-010-1975-8

Price, R. B., Kuckertz, J. M., Siegle, G. J., Ladouceur, C. D., Silk, J. S., Ryan, N. D., . . .y Amir, N. (2015). Empirical recommendations for improving the stability of the dot-probe task in clinical research. Psychological Assessment, 27(2), 365-376. https://doi. org/10.1037\%2Fpas0000036
Qureshi, A., Monk, R. L., Pennington, C. R., Wilcockson, T. D. y Heim, D. (2019). Alcohol-related attentional bias in a gaze contingency task: Comparing appetitive and non-appetitive cues. Addictive Behaviors, 90, 312-317. https://doi.org/10.1016/j.addbeh.2018.11.034

Reeve, W. V. y Schandler, S. L. (2001). Frontal lobe functioning in adolescents with attention deficit hyperactivity disorder. Adolescence, 36, 749-765.

Robalino Guerra, P. E. y Musso, M. F. (2018). Inatención del conductor: un estudio acerca de las relaciones entre redes atencionales y la propensión a cometer errores durante la conducción. Interdisciplinaria, Revista de Psicología y Ciencias Afines, 35(2), 425-444.

Robinson, T. E. y Berridge, K. C. (1993). The neural basis of drug craving: An incentive-sensitization theory of addiction. Brain Research Reviews, 18, 247-291. https://doi. org/10.1016/0165-0173(93)90013-p

Rosa, P. J., Esteves, F. y Arriaga, P. (2015). Beyond Traditional Clinical Measurements for Screening Fears and Phobias. Instrumentation and Measurement, IEEE Transactions on Instrumentation and Measurement, 64(12), 3396-3404. https://doi.org/10.1109/ TIM.2015.2450292

Roselló, J. (1998). Psicología de la atención. Introducción al estudio del mecanismo atencional. Madrid: Pirámide.

Sánchez, A. y Vázquez, C. (2012). Sesgos de atención selectiva como factor de mantenimiento y vulnerabilidad a la depresión: Una revisión crítica. Terapia Psicológica, 30, 101-115. https://doi.org/10.4067/S071848082012000300010

Sánchez-López, A., Quinto-Guillen, R., PérezLucas, J., Jurado-Barba, R., Martínez-Grass, I., Ponce-Alfaro, G. y Rubio-Valladolid, G. (2015). Validación de la versión española del Test Stroop de Alcohol. Anales de Psicología, 31(2), 504-523. https://doi.org/10.6018/ analesps.31.2.167491

Schmitz, F. y Svaldi, J. (2017). Effects of Bias Modification Training in Binge Eating Disorder. Behavior Therapy, 48(5), 707-717. 
https://doi.org/10.1016/j.beth.2017.04.003

Schoenmakers, T. M., de Bruinc, M., Lux, I. F., Goertz, A. G., Van Kerkhof, D. H. y Wiers, R. W. (2010). Clinical effectiveness of attentional bias modification training in abstinent alcoholic patients. Drug and Alcohol Dependence, 109, 30-36. https://doi.org/10.1016/j.drugalcdep.2009.11.022

Stacy, A. y Wiers, R. (2010). Implicit cognition and addiction: a tool for explaining paradoxical behavior. Annual Review of Clinical Psychology, 6, 551-575. https://doi. org/10.1146/annurev.clinpsy.121208.131444

Stroop, J. R. (1935). Studies of interference in serial verbal reactions. Journal of Experimental Psychology, 18(6), 643-662. https:// doi.org/10.1037/h0054651

Torres-Salazar, C., Moreta-Herrera, R., RamosRamírez, M. y López-Castro, J. (2020). Cognitive Bias of Optimism and Perception of Wellbeing in a Sample of Ecuadorian University Students. Revista Colombiana de Psicología, 29(1), 61-72. https://doi.org/10.15446/rcp. v29n1.7585

Treisman, A. (1969). Strategies and models of selective attention. Psychological Review, 76(3), 282-299. https://doi.org/10.1037/ h0027242

Vadhan, N., Carpenter, K., Copersino, M., Hart, C., Foltin, R. y Nunes, E. (2007). Attentional bias towards cocaine-related stimuli: relationship to treatment-seeking for cocaine dependence. American Journal of Drug and Alcohol Abuse, 33, 727-736. https://doi. org/10.1080/00952990701523722

Wadlinger, H. A. e Isaacowitz, D. M. (2010). Fixing our focus: Training attention to regulate emotion. Personality and Social Psychology Review, 15, 75-102. https://doi. org/10.1177\%2F1088868310365565

Waters, A. J. y Feyerabend, C. (2000). Determinants and effects of attentional bias in smokers. Psychology of Addictive Behaviors, 14, 111-120. https://doi.org/10.1037/0893164X.14.2.111
Weinstein, A. y Cox, W. (2006). Cognitive processing of drug-related stimuli: the role of memory and attention. Journal of Psychopharmacology, 20, 850-859. https://doi. org/10.1177/0269881106061116

Wiers, C. E., Stelzel, C., Gladwin, T. E., Park, S. Q., Pawelczack, S., Gawron, C. K., ... y Lindenmeyer, J. (2015). Effects of cognitive bias modification training on neural alcohol cue reactivity in alcohol dependence. American Journal of Psychiatry, 172(4), 335-343. https:// doi.org/10.1176/appi.ajp.2014.13111495

Wiers, R. W., Gladwin, T. E., Hofmann, W., Salemink, E. y Ridderinkhof, K. R. (2013). Cognitive Bias Modification and Control Training in Addiction and Related Psychopathology: Mechanisms, Clinical Perspectives and Ways Forward. Clinical Psychological Science, 1(2), 192-212. https://doi. org/10.1177/2167702612466547

Wiers, R., Bartholow, B. y van den Wildenberg, E. (2007). Automatic and controlled processes and the development of addictive behaviors in adolescents: a review and a model. Pharmacology Biochemestry Behaviors, 86, 263-283. https://doi.org/10.1016/j.pbb.2006.09.021

Wiers, R. W., Houben, K., Fadardi, J. S., van Beek, P., Rhemtulla, M. y Cox, W. M. (2015). Alcohol Cognitive Bias Modification training for problem drinkers over the web. Addictive Behavoirs, 40, 21-26. https://doi.org/10.1016/j. addbeh.2014.08.010

Yeung, E. S. y Sharpe, L. (2019). Cognitive Bias Modification for Social Anxiety: The Differential Impact of Modifying Attentional and/ or Interpretation Bias. Cognitive Therapy and Research, 43(4), 781-791. https://doi. org/10.1007/s10608-019-10012-3

Zack, M., Belsito, L., Scher, R., Eissenberg, T. y Corrigall, W. (2001). Effects of abstinence and smoking on information processing in adolescent smokers. Psychopharmacology, 153, 249-257. https://doi.org/10.1007/ s002130000552 